apuntesuniversitarios.upeu.edu.pe

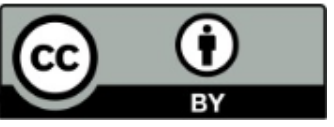

Apuntes Universitarios, 2020: 10(3), julio-setiembre

ISSN: 2304-0335 DOI: https://doi.org/10.17162/au.v10i3.475

\title{
El respeto: un valor que desafía a la educación de universitarios tabasqueños en la modernidad liquida
}

\section{Respect: a value that challenges the education of tabasquean university students in liquid modernity}

Gladys Hernández Romero ${ }^{1 \mathrm{a}}$

Universidad Juárez Autónoma de Tabasco, México ${ }^{1}$

(iD) ORCID: https://orcid.org/0000-0002-9045-29371

Recibido: 01 de noviembre de 2019

Aceptado: 01 de junio de 2020

\section{Resumen}

Los valores son considerados una guía o patrón de comportamiento que orientan los modos particulares de ser y vivir del individuo. Además, forman el criterio de juicio, preferencia y elección que conducen a una determinada acción. En este sentido, el objetivo de esta investigación es conocer la práctica de valores durante la formación profesional de los universitarios, priorizando el respeto. Para la recolección de datos se aplicó un cuestionario de elaboración propia de ocho ítems a muestra intencional de 100 estudiantes, con edades que oscilaron entre los 18 y los 20 años, dentro de una universidad pública ubicada al sur-sureste de los Estados Unidos Mexicanos. Los resultados señalan que los participantes consideran practicar los valores; sin embargo, estas respuestas llaman poderosamente la atención, ya que en contraparte manifiestan la carencia en prácticas éticas en la convivencia diaria dentro de la institución académica. Dentro de las conclusiones se puntualiza la necesidad de establecer acciones que aminoren la presencia de conflictos en la vida académica y relaciones profesionales, formando así un entorno de ética para la futura generación laboral.

Palabras clave: educación ciudadana, modernidad líquida, comportamiento social, respeto, valores, ética.

\begin{abstract}
Values are considered a guide or pattern of behavior that guides the particular ways of being and living of the individual. Furthermore, they form the criteria of judgment, preference and choice that lead to a certain action. In this sense, the objective of this research is to know the practice of values during the professional training of university students, prioritizing respect. For data collection, a
\end{abstract}

${ }^{\mathrm{a}}$ Correspondencia al autor:

E-mail: gladiolita6@ hotmail.com 
self-made questionnaire of eight items was applied to an intentional sample of 100 students, with ages ranging from 18 to 20 years old, within a public university located in the south-southeast of the United Mexican States. The results indicate that the participants consider practicing the values; however, these outcomes powerfully attract attention, since in counterpart they manifest the lack of ethical practices in daily coexistence within the academic institution. Among the conclusions, the need to establish actions that reduce the presence of conflicts in academic life and professional relationships is pointed out, thus forming an ethical environment for the future generation of work.

Keywords: citizenship education, liquid modernity, social behavior, respect, values, ethics.

\section{Introducción}

La cultura de la posmodernidad, llamada por Bauman modernidad líquida, se caracteriza por la fluidez incesante. No existe tal estado como el sólido. Bauman toma el adjetivo de las ciencias naturales, al considerar la similitud que existe entre las propiedades de los gases y los líquidos con la sociedad actual. 'Los fluidos, por así decirlo, no se fijan al espacio ni se atan al tiempo" (Bauman, 2000, p. 8); los sólidos, por lo contrario, se apegan a su estructura, conservan su forma ante el paso del tiempo y permanecen estáticos. De esta manera él desarrolla este concepto y muestra cómo los rasgos de nuestra sociedad coinciden con los del mencionado estado de la materia.

En esta sociedad, pues, ninguna instancia escapa al cambio. Esta disolución de esquemas rígidos, que comenzó por las estructuras económicas y financieras, pronto permeó el campo de la política y llegó a todas las instituciones. Ahora, toca a la esfera de lo privado. El hombre también experimenta esta falta de consistencia, de firmeza, en su día a día. Y en medio de este cambio vertiginoso que, como ya se mencionó, ha llegado a lo privado de nuestras vidas, se inscriben los vaivenes de la ética.

Hablar de valores en universitarios es entrar en un tema con ciertas aristas ya que, lo que los jóvenes generalmente tienen, es su propio concepto de lo que significan. Los valores son los principios y cualidades que caracterizan a una persona, e impulsan a los individuos a actuar de acuerdo con sus creencias, reflejar su conducta, y expresar sus intereses y sentimientos. En este caso, Munguía (2015) considera que un valor es un atributo que todo ser humano tiene sobre su entorno incluyendo personas, objetos o actos; es decir, las actitudes personales que influyen en el reconocimiento de toda cosa primordial para el sujeto y que impactan directamente en la toma de decisiones. 
Actualmente, es evidente una gran ausencia de valores sociales y morales en la sociedad, y “(...) estas conductas son antisociales y deshumanizadas, pero desafortunadamente cada día que pasa se están instalando más dentro de la sociedad actual" (Acosta, 2018), sobre todo en los estudiantes universitarios, quienes se están formando para la vida profesional, y que finalmente son producto del sistema en la que viven.

Entre los principales valores ausentes se encuentra el respeto, el cual bien puede ser la madre de todas las virtudes (Piscoya, (2014). Consiste en reconocer las cualidades, méritos, situación y valores particulares de toda persona aún si existe una contraposición de pensamie nto con el sujeto mismo (Negrete, 2014). En un estudio sobre la actual crisis moral y de valores en México, Herrera-Gutiérrez y Torres (2017) señalan el descuido ante la degradación de los mismos y el deterioro del tejido social:

(...) la educación en valores de un grupo social es fundamental ya que es la base para el individuo que le permite enfrentar lo que venga en su vida desde una doble perspectiva, la del sentido de su propia vida y la de la convivencia humana. (p.3)

Y es que los valores son la base para una sana convivencia en la sociedad, por lo que merecen la importancia necesaria para que las generaciones futuras sigan promoviendo su aplicación y enseñanza. Ante esta idea, Macías y Suárez (2015) apuntan que es prioritario hacer hincapié en el reto que implica su desarrollo en los seres humanos y la importancia de practicarlos en la cotidianidad y comparten que el respeto es una de las acciones básicas para convivir con los demás; no obstante, este valor empieza por uno mismo, el poseer auto respeto y amor propio conduce a ser más justo con los demás.

González y Cardentey (2016) estiman que los valores son tan importantes en la vida estudiantil universitaria que deben ser contextualizados y dirigidos hacia cada uno de los alumnos matriculados, ya que están impresos en sus formas de actuar. La formación profesional exenta de práctica ética es un tema detonante que puede perjudicar el bienestar futuro de la sociedad, así como el campo académico y laboral.

Hablar de los valores, es hablar de una estructura en la que entran en juego pensamiento y sentimiento como requisitos previos para mover la voluntad y la acción. (Chapa y Martínez, 2015). Baxter (2009) plantea la idea de educar en valores como el hecho orientado y planificado que tiene tanto la familia como la escuela en la responsabilidad de formar y dirigir las nuevas generaciones. "Cumplir, ser paciente, dedicado, entusiasta, perseverante son características que se asignan al 
profesor (...)" (Navia y Hirsch, 2015, p.128) por lo que el docente juega un rol importante en la trasmisión de los valores hacia los alumnos. Es importante, por tanto, que las universidades divulguen los valores hacia sus alumnos, maestros y personas en general; pero más importante es difundirlos a los estudiantes, ya que ellos son la clave para llevarlos a la acción en sociedad de la manera correcta y adecuada y así tener una sana convivencia en todos los entornos de su vida.

Por otro lado, durante el proceso de aprendizaje en estudiantes universitarios es importante la práctica de valores. Para fines de esta investigación se priorizará el respeto, recordando que respetar es conocer límites y tomar en cuenta el entorno, así como reconocer que el interlocutor también puede tener razón, además, se debe determinar el radio de acción al que tiene derecho cada uno de los involucrados en la escena. Ante esto, Buitrago, Cubillos, Ramírez y Tabares (2011) afirman:

Está visto que el respeto lo aplicamos en todo momento, en todos los ámbitos de nuestra vida, sin este valor viviríamos en conflicto, todos tenemos derecho a ser respetados, y estamos o deberíamos estar obligados a dar ese mismo respeto a los demás. (p. 26).

Como tal, el respeto se encuentra dentro del código de ética de la institución de educación superior en la que se realizó este trabajo (UJAT, 2014), el cual estipula que "la comunidad universitaria reconoce los principios y valores fundamentales para el cumplimiento de la misión y objetivos institucionales, con su práctica se propicia un clima más agradable, productivo y transparente" (p. 5).

Dentro de este código se localizan los valores que dicha comunidad universitaria reconoce, los cuales son: honradez, transparencia, rendición de cuentas, respeto, lealtad, responsabilidad, competencia, efectividad y eficiencia, manejo de recursos, calidad personal, valor civil, la probidad y el diálogo (UJAT, 2014).

Entonces se puede decir que la comunidad estudiantil conoce en qué consisten los valores y principios; sin embargo, podría no estar empleándolos, lo que señala una situación preocupante, por lo que es vital incorporar los valores en los distintos niveles. Elexpuru, Villardón y Yániz, (2011) apuntan que es en el nivel superior donde estos valores y principios se convierten en un fundamental componente para lograr la formación integral y sólida que va a beneficiar a la sociedad.

En el ámbito de la educación, tanto los docentes como los directivos tienen como labor fundamental formar a estudiantes íntegros, responsables con su entorno, los guías deben ser capaces de despertar el interés del aprendizaje, de utilizar estrategias didácticas y tecnológicas para 
contar con profesionistas competitivos y asimismo armonizar la convivencia para favorecer el desarrollo emocional y social del alumno.

En este sentido, Ojalvo (2017) afirma que no basta con un proceso educativo universitario centrado únicamente en adquirir el conocimiento, pues es constante y de mayor crecimiento el interés en las instituciones por contribuir a formar profesionales con valores, que les permitan, no solamente convivir en el mundo actual, sino comprometerse a la transformación, a partir de su crecimiento como seres humanos. En opinión de Hirsh (2010):

Muchas universidades (...) han generado proyectos de investigación de largo plazo para conocer la situación de los estudiantes, profesores y egresados y han ido incorporando una formación explicita y sistemática, principalmente a partir de la introducción de materias obligatorias (p. 34).

Es en el caso de la institución de educación superior motivo de este trabajo, la asignatura de "Ética" es impartida en todas las licenciaturas disponibles, en las modalidades presencial y a distancia. Por otro lado, en referencia a la formación de los estudiantes, Mederos (2014) señala que 'El escenario de aprendizaje debe caracterizarse por el respeto, lo que facilita enseñar y aprender, por las buenas relaciones e interacciones entre todos los miembros de la comunidad educativa" (p. 142).

Por ello, en un mundo tan moderno y lleno de avances tecnológicos como el de hoy en día, surge la falta de valores y sobre todo del respeto en el ámbito escolar y familiar (Duarte y Abreu, 2013). Durante los últimos años se ha hecho evidente la decadencia en la cortesía y en los estándares éticos. La sociedad humana está atravesando una crisis de valores, lo que trae como consecuencia la insensibilidad y la falta de justicia e igualdad.

El respeto es un tema que cada día va cobrando mayor interés en el mundo moderno, tanto que esta palabra ha dejado de ser un misterio para introducirse en el vocabulario de toda persona, su poca formación en el seno familiar genera como consecuencia graves conflic tos en los contextos en los que se interactúa, con una latente provocación de situaciones violentas. (Uranga, Rentería y González, 2016, p. 188)

En este aspecto, si una persona carece del acercamiento hacia la ética como praxis humana puede alterar de forma negativa la interacción con su entorno. Al respecto, Chaparro y Gordon (2010) afirman: "El respeto crea un ambiente de seguridad y cordialidad; permite la aceptación de las limitaciones ajenas y el reconocimiento de las virtudes de los demás. Evita las ofensas y las ironías; no deja que la violencia se convierta en el medio para imponer criterios. (p.16). Y es que 
el respeto abarca múltiples dimensiones del ser humano, en su forma de ser, hacer, pensar y de relacionarse, incluso abarca las creencias, las costumbres, las cuestiones políticas y familiares, las cuales forman parte de la convicción personal, por lo cual la educación en valores es fundamental para las instituciones educativas con objetivo de formación integral. (Pérez, 2019).

Los estudiantes necesitan aceptar la diversidad, mantener posturas que les permitan progresar como individuos. Duarte y Abreu (2013) señalan que la autenticidad y el respeto deben estar siempre presentes en la vida del estudiante, quien debe desarrollar la capacidad de plantear sus inquietudes y sus problemas y por supuesto compartir las responsabilidades de las actividades expuestas y explicadas en clase. El objetivo de esta investigación es conocer la práctica de valores durante la formación profesional de los universitarios, priorizando el respeto.

\section{Método y materiales}

Este trabajo fue desarrollado en la división académica de Ciencias EconómicoAdministrativas, una de las 12 divisiones con la que cuenta la Universidad Juárez Autónoma de Tabasco, se encuentra ubicada en el sur-sureste de los Estados Unidos Mexicanos. Como parte de la investigación se aplicaron 100 cuestionarios a estudiantes pertenecientes a esta institución de educación superior con el objetivo de conocer la práctica de valores durante la formación profesional.

El tipo de investigación es cuantitativa, y se caracteriza por la selección subjetiva e intersubjetiva de indicadores - a través de conceptos y variables-de ciertos elementos de procesos, hechos, estructuras y personas (Cadena-Iñiguez et. al., 2017). El estudio se basó en una muestra intencional o de conveniencia de cien estudiantes de primero y quinto semestre de las 4 carreras con que cuenta dicha División: Administración, Contabilidad, Economía y Mercadotecnia. Se eligieron dichos semestres porque los estudiantes recién ingresados pertenecían a ese nivel académico en la institución $\left(1^{\circ}\right)$ y quienes están a la mitad de la licenciatura $\left(5^{\circ}\right)$. El criterio para determinar la muestra se basó en la elección de estudiantes regulares quienes, al momento de administrar los cuestionarios de elaboración propia, cursaban sus estudios en la modalidad presencial, no tomándose en cuenta el turno al que asisten, su avance curricular (a pesar de cursar el quinto semestre, el avance curricular es variado, ya que esta institución de educación superior tiene el modelo curricular flexible), ni su estado civil. 
Para la recolección de datos se procedió a aplicar un cuestionario con opción de respuesta múltiple a cien estudiantes; este consta de ocho preguntas obteniéndose una confiabilidad de 0.74 (alfa de Cronbach), que para Hall (1963) supera el valor mínimo recomendado de .7. En este cuestionario, se hizo una serie de preguntas acerca de los valores y la frecuencia con que los estudiantes los practican con los padres, amigos, en la universidad y vecinos, además del valor que debe tener un universitario y con cuál se identifica. En cuanto a la validación se envió a expertos a fin que evaluaran redacción y descartar errores o confusiones (Pérez, et al. 2006; Vázquez. 2010; Ahani, et al., 2012); por lo que se proporcionó un juego con las cuestiones, a cinco profesores expertos en la materia y se le leyó a cada uno, por lo que se hicieron los ajustes necesarios, de acuerdo a sus sugerencias. Los informantes, 25 de cada una de las mencionadas licenciaturas accedieron a responder las preguntas planteadas.

\section{Resultados y discusión}

En los resultados obtenidos del cuestionario de elaboración propia que se aplicó a los cien estudiantes, se obtuvo que el $40 \%$ de los participantes pertenece al género masculino, mientras que el $60 \%$ al femenino. Sus edades fluctúan entre los 17 y los 31 años, teniendo un rango de 14. El $50 \%$ cursa el primer semestre de su carrera y el otro $50 \%$ quinto semestre.

A pesar que el instrumento se aplicó solamente a alumnos de semestres seleccionados, permite analizar la práctica de los valores y su actual rezago en los estudiantes universitarios. De aquí la importancia de su práctica y, sobre todo, tenerlos presentes en la formación profesional con el fin de mantenerse en un mundo cada día más competitivo, puesto que ayudan al estudiante a desempeñarse como una mejor persona en todos los ámbitos de su vida y son un factor clave para alcanzar el éxito.

Las licenciaturas a las que pertenecen los estudiantes son: Administración, Contabilidad, Economía y Mercadotecnia, ocupando un porcentaje de participación del 25\% cada una, en esta investigación. La tabla 1 muestra la frecuencia con la que practican los valores con sus padres, amigos, profesores o vecinos, y entre compañeros. 
Tabla 1. Frecuencia práctica de los valores

\begin{tabular}{lll}
\hline & Frecuencia & Porcentaje \\
\hline $\begin{array}{l}\text { Con padres, amigos, } \\
\text { profesores o vecinos }\end{array}$ & Siempre & 61 \\
& Casi siempre & 36 \\
& Pocas veces & 3 \\
& Nunca & 0 \\
Entre compañeros & Siempre & 44 \\
& Casi siempre & 39 \\
& Pocas veces & 15 \\
& Nunca & 2 \\
\hline
\end{tabular}

Fuente: elaboración propia

Con respecto al lugar donde consideran que se forjan los valores de los universitarios, la mayoría (83\%) respondió que en el hogar, mientras que el 15\% considera que es en la escuela, un $1 \%$ contestó que con los amigos y el otro $1 \%$ considera que en la religión. Ante esto, Garrido et al. (2014) concuerdan con una perspectiva similar señalando a la familia como "la primera institución que ejerce influencia en el niño mediante la transmisión de valores, costumbres y creencias por medio de la convivencia diaria; es la primera institución educativa y socializadora del niño" (p. 149). En la tabla 2 se muestra la frecuencia con la que hablan con sus amigos sobre el respeto hacia los padres y profesores, en ambos casos domina el porcentaje correspondiente al $42 \%$, lo que determina que pocas veces conversan con sus compañeros sobre el tema.

Tabla 2. Habla con sus amigos sobre el respeto

\begin{tabular}{lll}
\hline & Frecuencia & Porcentaje \\
\hline Hacia sus padres & Siempre & 12 \\
& Casi siempre & 37 \\
& Pocas veces & 41 \\
Hacia sus profesores & Nunca & 10 \\
& Siempre & 13 \\
& Casi siempre & 37 \\
& Pocas veces & 42 \\
& Nunca & 8 \\
\hline
\end{tabular}

Fuente: elaboración propia 
En estas condiciones, ¿qué hacer en una sociedad en la que la violencia parece haberse entronizado? Como muestra, se presentan algunos datos: Según cifras oficiales de la Encuesta Nacional de Victimización y Percepción sobre Seguridad Pública, realizada por el Instituto Nacional de Estadística y Geografía (INEGI) se estima que en 2017 el número de víctimas en México ascendió a 25.4 millones de personas de 18 años y más. El 35.6\% de los hogares mexicanos contó con al menos una víctima de algún tipo de delito. En la figura 1 se observa la frecuencia con que saludan a vecinos, profesores o amigos para darles los buenos días, tardes o noches, un $47 \%$ señala que siempre, el $40 \%$ respondió que casi siempre, un 12\% eligió pocas veces y un 1\% señaló que nunca.

\begin{tabular}{|l|l|}
\hline \multicolumn{1}{|c|}{ Frecuencia } & \\
\hline Siempre & $47 \%$ \\
\hline Casi siempre & $40 \%$ \\
\hline Pocas veces & $12 \%$ \\
\hline Nunca & $1 \%$ \\
\hline
\end{tabular}

Figura 1. Frecuencia de saludar a sus vecinos, profesores o amigos. Fuente: elaboración propia.

Como se mencionó anteriormente, el respeto es una práctica primordial para el ser humano, es el valor que los padres enseñan a sus hijos para ser buenas personas, el respeto hacia los demás y hacia uno mismo. Y es que, como valor, abre el camino a la convivencia pacífica, a la aceptación a la diversidad y vigoriza la tolerancia. Ya los antiguos griegos se ocuparon de la importancia de mantener claridad en los límites de las relaciones, si es que se quiere alcanzar el bien común. Pero en la sociedad globalizada, estos parámetros han sucumbido ante las exigencias de libertad y de individualidad. Tal como se observa en la tabla 3 , los resultados a la pregunta si consideran que los estudiantes ponen en práctica los valores dentro y fuera de la institución muestra lo siguiente. 
Tabla 3. Ponen en práctica los valores

\begin{tabular}{lll}
\hline & Frecuencia & Porcentaje \\
\hline Dentro de la Institución & Siempre & 10 \\
& Casi siempre & 37 \\
& Pocas veces & 48 \\
& Nunca & 5 \\
Fuera de la Institución & Siempre & 7 \\
& Casi siempre & 40 \\
& Pocas veces & 50 \\
& Nunca & 3 \\
\hline
\end{tabular}

Fuente: elaboración propia

Aunque el valor preponderante fue el respeto, en ocasiones no es practicado de la forma que se espera; por ello, de acuerdo con la opinión de los participantes, en pocas ocasiones los universitarios demuestran poner en práctica sus valores dentro de la institución. Al respecto, Dolan (2019) señala que "Los valores son creencias arraigadas sobre lo que está bien, lo que está correcto y apropiado y nos ayudan a modelar nuestro carácter y forma de actuar si queremos ser valiosos para nosotros mismos y útiles para la sociedad" (p.19) y para la mayoría de los participantes en este estudio, los universitarios actualmente han perdido el rumbo en este sentido.

Otro de los puntos críticos en México, como en muchos otros países, es la violencia contra las mujeres. En 2007 se promulgó la Ley General de Acceso a las Mujeres a una Vida Libre de Violencia, la cual ha sido un referente fundamental en la materia. No obstante, una de cada tres mujeres mayores de 14 años reconoce haber sufrido violencia física en su infancia, según la Encuesta Nacional sobre la Dinámica de las Relaciones en los Hogares (ENDIREH) realizada en 2016 y publicada en Violencia y feminicidio en niñas y adolescentes en México. (ONU, 2018). Según el mismo estudio, en 2017, el 3.1\% de las defunciones de niñas de 0 a 4 años, fue ocasionada por violencia familiar.

Díaz, Gómez y Melo (2016) comparten que: "En un mundo en constante cambio y con necesidades insatisfechas cada vez mayores, es imprescindible contar con profesionales idóneos, no solo en el campo académico - técnico, sino principalmente en el de valores" (p.2), y resaltan el interés de formar alumnos capaces de reflejar buenas prácticas, pues de ello resultará el fruto del progreso comunitario. La formación en valores es una necesidad fundamental que las instituciones educativas deben afrontar hoy. Es común escuchar quejas sobre las limitaciones de la escuela, sobre 
la falta de estructura familiar, la tiranía del mercado que impone el consumismo y demás problemas; no obstante, como diría Camps, es hora de creer en la educación.

Si apuntamos sistemáticamente a la educación ante cualquier situación conflictivamaltrato de la mujer, violencia callejera, vandalismo, falta de civismo - es porque no todo se resuelve legislando. Las leyes no transforman la realidad si no van acompañadas de la voluntad de las personas que, en definitiva, tienen que aplicar las leyes y hacerlas cumplir correctamente. Crear personas responsables es una de las finalidades de la educación (2008, p.16).

Por su parte, Hitchman, Machado y Panol (2012) determinan que:

El sistema de valores está directamente vinculado por la forma de la sociedad, lo que asegura que al cambiar las relaciones sociales, y sobre todo las económicas, se establezcan nuevos valores, y que tenga una tenaz lucha ideológica, por lo que el maestro debe tener en cuenta en sus clases hacer consciente a sus estudiantes de lo correcto y lo incorrecto en cada una de sus acciones (p. 428).

De esta forma, la ausencia de valores-específicamente, el respeto-entre los jóvenes, es un problema complejo, multifactorial, que se relaciona con aspectos tales como la violencia intrafamiliar, la situación económica y el nivel educativo de una sociedad. Por tanto, el docente tiene la responsabilidad de contribuir en esta tarea de promoción de valores éticos, pero no es el único actor, y no podrá, en consecuencia, operar un verdadero cambio, mientras las estructuras sociales de desigualdad y violencia continúen vigentes.

\section{Conclusiones}

Luego de haber analizado los resultados del cuestionario aplicado a los estudiantes de esta universidad, se puede concluir que la mayor parte de ellos se identifica con el valor "respeto", el mismo que es considerado por diversos autores como uno de los principales en la vida de todo ser humano y el cual debe practicarse en todo momento para una sana convivencia con la sociedad.

Aunque los resultados arrojan una postura cercana a la práctica de este valor, es elemental señalar que esto no se refleja con el porcentaje de prácticas éticas en los universitarios, pues la respuesta de gran parte de ellos evidencia un espacio de duda en aplicarlos de forma continua.

Se vive así en tiempos en los que se pretende que todo se permita, si es que corresponde al bienestar personal. A la vez, los niveles de intolerancia se agudizan, dando un panorama por demás complejo de una sociedad fragmentada y que vive, desde ambos extremos, las consecuencias al enfrentar elevados índices de violencia. Esto produce una sociedad urgida de la intervención activa 
y efectiva de sus instituciones, lo que demuestra que no está de más promover el respeto en los estudiantes universitarios, ya que ellos son los que llevan en sus manos el camino hacia una vida profesional exitosa. Por el contrario, si no hay respeto, se carece de todos los demás valores, por lo que hace a un individuo pobre en su persona.

Ser una persona con valores tiene como consecuencia que se abran muchas puertas en la vida, oportunidades que no a cualquiera se le presentan ya que tenerlos y practicarlos realmente, habla bien de uno mismo y puede ser el ejemplo o la inspiración de alguien más. El éxito no se obtiene solamente con inteligencia y trabajo arduo, con valores es mayor la posibilidad de conseguirlo.

Los jóvenes necesitan unir esfuerzos, reforzar ideas y establecer planes de acción para un beneficio mutuo. Es urgente que los estudiantes se respeten a sí mismos y se proyecten en el futuro, de aceptar la idiosincrasia de los demás, de comunicarse correctamente, de no dañar la integridad física y emocional de sus compañeros, se debe valorar el cumplimiento de las normas y reforzarlas con mayor autonomía y todos los agentes sociales deben ser dialogantes, firmes, afectivos y éticos.

Por tanto, el presente trabajo se ocupa de la educación, pero es claro que no se trata de proyectos parcelados, sino que todos los órdenes sociales deberían integrarse en un todo que busque romper con este esquema. No es el objetivo de este trabajo dar cuenta de la inseguridad en el país, sólo se han mencionado dos de los graves problemas que aquejan a nuestra sociedad, para ubicar en contexto el papel de la educación.

\section{Referencias}

Acosta, C. (2018). Crisis de valores en la sociedad actual Recuperado de https://fundacionministeriodefamilia.webnode.es/_files/200000339_ 12919138a7/Crisis\%20de\%20valores\%20en\%201a\%20sociedad\%20actual.pdf

Ahani, M., Bahrami, H. R., \& Rostami, M. (2012). Determining and ranking dimensions of knowledge management implementation using Hicks model and fuzzy TOPSIS Technique. Management Science Letters, 721-730. Recuperado de http:/www.econbiz.de/Record/determining-and-ranking-dimensions-of-knowled gemanagement-implementation-using-hick s- model-and-fuzzy-topsis-technique-ahanimona/10009720861.

Bauman, Z. (2000). Modernidad Líquida. México: Fondo de Cultura Económica

Buitrago, E., Cubillos, F., Ramírez, J, y Tabares, J. C. (2011). Estrategias personalizantes para fortalecer el valor del respeto en las relaciones interpersonales en los educandos del grado 
noveno. "A" de la Institución Educativa Gabriela Mistral. Recuperado de http://repositorio.ucm.edu.co:8080/jspui/bitstream/handle/10839/177/Esther\%20Buitrago\% 20G.pdf;jsessionid=8382289C100CE168DD87BAD6DCCCB4FC? sequence $=1$

Cadena-Iñiguez, P., Rendón-Medel, R., Aguilar-Ávila, J., Salinas-Cruz, E., de la Cruz-Morales, F. R. y Sangerman-Jarquín, D. M. (2017). Métodos cuantitativos, métodos cualitativos o su combinación en la investigación: un acercamiento en las ciencias sociales. Revista Mexicana de Ciencias Agrícolas, 8 (7), 1603-1617. Recuperado de https://www.redalyc.org/pdf/2631/263153520009.pdf

Camps, V. (2008). Creer en la Educación. Barcelona: Península.

Castillo, V., Aguilar, J., Robles, R., Sánchez, C., Ábrego, Y. y Batista, L. (2017). Valores universitarios y profesionales de los estudiantes de postgrado de la Universidad Tecnológica de Panamá. Revista de Iniciación científica, 3 (1), 14-20. Recuperado dehttps:/www.google.com.mx/url?sa=t\&source=web\&rct=j\&url=http://revistas.utp.ac.pa/i ndex.php/ric/article/download/1693/2429\&ved=2ahUKEwj8kf6k4rraAhVCuVkKHZeNAS gQFjAFegQIARAB\&usg=AOvVaw0KtbQd1BMvRaZBwQ0IOIhj

Chapa, P. y Martínez, T. (2015). Valores Universitarios en los Jóvenes Estudiantes de la Facultad de Ciencias Políticas y Administración Pública, UANL. Revista Iberoamericana para la investigación y el Desarrollo Educativo, 6(11), 773-786. Recuperado de: http:/www.redalyc.org/html/4981/498150319046/

Chaparro, R. A. y Gordon, M. S. (2010). Fortalecimiento del valor del respeto como estrategia para promover la convivencia con los padres de familia del grado de transición de la institución educativa García Márquez. $\quad$ Recuperado de http://observatorio.unillanos.edu.co/portal/archivos/10PROYECTOFORTALECERELRES PETO.pdf

Díaz, A. R., Gómez, E. y Melo, A. (2016). La importancia de la educación en valores éticos para la administración pública: un estudio exploratorio. Gestión Estratégica: de la crisis a la oportunidad. Conferencia llevada a cabo en el Congreso Internacional de Administración, Brasil

Dolan, S. (2019). Más coaching por valores. Madrid: LID

Duarte, A. y Abreu, J. (2013). El respeto como identidad del docente; una comparación entre puntos de vista de alumnos y docentes. Red de Investigación Educativa, 908-927. Recuperado de: https://www.google.com.mx/url?sa=t\&source=web\&rct=j\&url=http://eprints. uanl.mx/8026 /1/a1_2.pdf\&ved=2ahUKEwicz7a97braAhXlp1kKHcH9AxcQFjAAegQICBAB\&us g=AO vVaw05LX3XVC3YCiUGty7Lr6eJ

Elexpuru, I., Villardón, L. y Yániz, C. (2011). Identificación y desarrollo de valores en estudiantes universitarios. Revista de Educación, Publicación anticipada en línea. DOI: 10-4438/1988592X-RE-2011-362-158 
Garrido, A., Reyes, A., Ortega, P. y Torres, L. E. (2014). La disciplina y los valores de jóvenes universitarios. Alternativas en psicología, (30), 147-160. Recuperado de http://www.alternativas.me/attachments/article/61/10\%2520\%2520La\%2520disciplina\%25 20y52520los\%2520valores\%2520de\%2520j\%25C3\%25B3venes\%2520universitarios.pdf

Gómez, L., Díaz, S. B., Barrios, C., Morejón, E. L. y Padrón, A. (2014). La educación en valores profesionales en la Filial de Tecnología de la Salud Simón Bolívar. Revista de Ciencias Médica, 18(1), 111-123. Recuperado de http://scielo.sld.cu/scielo.php?script=sci_arttext\&pid=S1561-31942014000100012

González, R. y Cardentey, J. (2016). Educación en valores de estudiantes universitarios. Humanidades médicas, 16(1), 161-174. Recuperado de http://scielo.sld.cu/pdf/hmc/v16n1/hmc11116.pdf

Hall, R. H. (1963). The concept of bureaucreacy: an empirical assessment. The American JournalofSociology, 69(1), 32-40. Recuperado de http://tarjomefa.com/wpcontent/uploads/2016/08/4726-English_2.pdf

Hernández, R. Y. (2019). Valores éticos y estéticos en la formación de los estudiantes de las ciencias médicas. Medisur, 17(2), 120-172. Recuperado de http://scielo.sld.cu/pdf/ms/v17n2/1727-897X-ms-17-02-170.pdf

Herrera-Gutiérrez, L. C. y Torres, R. G. (2017). Por qué la crisis moral y de valores en nuestro país. Congreso Nacional de Investigación Educativa- COMIE XIV, San Luis Potosí. Recuperado de http:/www.comie.org.mx/congreso/memoriaelectronica/v14/doc/1016.pdf

Hirsch, A. (2010). Ética profesional y profesores universitarios: una perspectiva comparativa. Reencuentro, 57, 34-38. Recuperado de https:/www.redalyc.org/pdf/340/34012514005.pdf

Hitchman, D., Machado, I., y Panol, M. (2012). La formación de valores de la juventud de nuestros días. Revista Cubana de Higiene y Epidemiología, 50(3), 427-437. Recuperado de http://scielo.sld.cu/scielo.php?script=sci_arttext\&pid=S1561$\underline{30032012000300018 \& \operatorname{lng}=\mathrm{es} \& \mathrm{t} \operatorname{lng}=\mathrm{es}}$

Macías, F. y Suárez, H. D. (2015). Un acercamiento de los docentes de la carrera de trabajo social a la educación en valores. Revista Médica de Santiago de Cuba-MEDISAN, 19(8), 968- 974. Recuperado de http://www.medisan.sld.cu/index.php/san/article/view/405

Mederos, M. (2014). La convivencia entre los estudiantes universitarios: su atención desde el proyecto educativo de la carrera. Revista Iberoamericana de Evaluación Educativa, 7(1), 141-159. Recuperado de https://revistas.uam.es/index.php/riee/article/view/3395/3610

Munguía, R. (2015). Ética y Valores 1. México. D.F.: Secretaría de Educación Pública.

Navia, C. y Hirsch, A. (2015). Dimensiones y rasgos sobre la excelencia del profesorado en instituciones formadoras de docentes en dos países de América Latina. EDETANIA 48, 117 130. Recuperado de https://revistas.ucv.es/index.php/Edetania/article/view/41/40 
Negrete, L. A. (2014). Valores universales. Universidad Veracruzana. Recuperado de https://www.uv.mx/psicologia/files/2014/11/VALORES-UNIVERSALES.pdf

Ojalvo, V. (2017). La educación de valores. Reflexiones y experiencias desde el enfoque históricocultural. Tarbiya, Revista de Investigación e Innovación Educativa, 30, 47-60. Recuperado de

https://revistas. uam.es/tarbiya/article/viewFile/7390/7711\&ved=2ahUK Ewj81sy2qpDcAh USQ6wKHQYNDFI4FBAWMAx6BAgGEAE\&usg=AOvVaw3wllZy5_PTwCpcxMAgL$\underline{\mathrm{JH}}$

Pérez, S., Montes, J. M., \& Vázquez, C. J. (2006). Human Resources Management As A Determining Factor In Organisational Learning. Management Learning, 37(2), 215-239. https://doi.org/10.1177/1350507606063443

Pérez, L. F. (2019). Consideraciones epistemológicas, psicológicas, sociológicas y pedagógicas de la educación en valores. Revista Iberoamericana para la Investigación y el Desarrollo Educativo, 9(18), 184-194. http//dx.doi.org/10.23913/ride.v9i18.417

Piscoya, D. A. (2014). Diferencias del nivel de formación de la honestidad en estudiantes de dos instituciones educativas de secundaria de Lambayeque-2014 (Tesis de maestría). Universidad Católica Santo Toribio de Mogrovejo, Chiclayo, Perú. Recuperado de http://tesis.usat.edu.pe/bitstream/20.500.12423/1160/1/TM_PiscoyaBarreraDemetrioAlbert o.pdf.pdf

Universidad Juárez Autónoma de Tabasco (2014). Código de ética de la Universidad Juárez Autónoma de Tabasco. México. Recuperado de http://www.archivos.ujat.mx/abogado_gral/legislacion_univ2012/CODIGO\%20DE\%20ETI CA\%20DE\%20LA\%20UNIVERSIDAD\%20JUAREZ\%20AUTONOMA\%20DE\%20TAB ASCO.pdf

Uranga, M. S., Rentería, D. E. y González, G. J. (2016). La práctica del valor del respeto en un grupo de quinto grado de educación primaria. Revista Ra Ximhai, 6(12), 187-204. Recuperado de http://www.redalyc.org/pdf/461/46148194012.pdf

Vásquez Rizo, F. E. (2010). Modelo de gestión del conocimiento para medir la capacidad productiva en grupos de investigación. Ciencia, docencia y tecnología, 41, 101-125. Recuperado de http:/www.scielo.org.ar/scielo.php?pid=S1851 $17162010000200005 \&$ script=sci_arttext. 